\title{
Zum Stand der Rechtsstaatlichkeit in Afghanistan
}

\author{
Von Parinas Parhisi, Frankfurt/Main*
}

\section{Einleitung}

In Afghanistan eine Justizreform und Rechtsstaatlichkeit zu etablieren, ist erklärtes Ziel der dort engagierten Staatengemeinschaft. Herstellung und Ausbau von Gerechtigkeit sind für einen modernen stabilen Staat unverzichtbar. Betrachtet man jedoch den Bereich der afghanischen Justiz, so fragt man sich, spätestens wenn man vor Ort arbeitet, ob und inwieweit die internationale Aufbauhilfe als effizient bezeichnet werden kann.

Eine ausdrückliche Garantie des Rechtsstaatsprinzips kennt die afghanische Verfassung nicht. Sie enthält allerdings zahlreiche mittelbare Bezüge, wie etwa Gewaltenteilung, Bindung von Exekutive und Judikative an Recht und Gesetz, ebenso wie die Garantie richterlicher Unabhängigkeit und die Gleichheit vor dem Gesetz. Ausführungen zum Verhältnismäßigkeitsprinzip von deutschen Ausbildern werden mit großem Interesse angenommen, ein ausgeprägter Sinn dafür ist aber noch nicht vorhanden. Dieser Beitrag intendiert, Problemfelder im Bereich der Justiz aus unmittelbaren, praxisnahen Erfahrungen mit afghanischen Richtern, Staatsanwälten und Polizisten zu skizzieren. Diese gehen auf eine Dozententätigkeit der Autorin im Auftrag des Heidelberger Max-Planck-Instituts für ausländisches öffentliches Recht und Völkerrecht zurück, welche im Rahmen des Justizaufbauprojektes in Afghanistan stattfand ${ }^{1}$. Die Verfasserin konnte bei mehrmaligen Aufenthalten (2007-2008) zahlreiche praktische Hindernisse im Umgang mit der Entwicklung des Rechtsstaates erfahren. Sie sollen im folgenden nachgezeichnet werden.

Die entscheidende Frage ist: Wie will der afghanische Staat, angesichts zunehmenden Terrors und wachsender Unsicherheit, den Vorgaben der Verfassung sowie den diesbezüglichen Zusicherungen in offiziellen Papieren der Staatengemeinschaft Bedeutung verschaffen, sie gleichsam in die Lebenswirklichkeit übersetzen? Bezogen auf den Rechtsstaatsaufbau ist zusätzlich von Bedeutung, ob islamische Komponenten der afghanischen Verfassung (AV) in Konkurrenz zu rechtsstaatlichen Vorgaben stehen. Mit anderen Worten: Ist Rechtsstaatlichkeit in einer Islamischen Republik möglich? Die Antwort auf diese komplexe Frage muss sehr differenziert ausfallen, was den Rahmen eines Berichts gewiss

Parinas Parhisi, Dr. jur., Lehrbeauftragte an der Johann-Wolfgang-Goethe-Universität Frankfurt/ M.; Referentin im Hessischen Ministerium der Justiz, für Integration und Europa. Die folgenden Ausführungen geben ausschließlich die Auffassung der Autorin wieder. E-mail: Parhisi@jur.unifrankfurt.de

1 Es handelt sich um mehrwöchige Dozenten-Aufenthalte in Kabul, Kunduz und Bamiyan. Allg. hierzu Stellungnahmen des MPI: http://www.mpil.de/ww/de/pub/forschung/forschung_im_detail/ glob_wisstransf/afghanistan_projekt/ber_2007.cfm; für einen Bericht, der mehr auf die Vorstellung des MPI-Projektes fokussiert ist: Parinas Parhisi, Aufbauhilfe für die Justiz: Richterausbildung in Afghanistan, in: JuS-Magazin, 3/2009, S. 18-19. 
sprengen würde. Nur einige wichtige Aspekte werden nachfolgend anhand ausgewählter Aspekte der praktischen Zusammenarbeit mit afghanischen Juristen dargestellt. Dies soll die vorhandenen Schwierigkeiten in der Entwicklung der rechtsstaatlichen Elemente in afghanischem Sektor näher beleuchten und zugleich Handreichungen für den Entwicklungsprozess geben.

\section{Verfassungsrechtliche Spannungsfelder}

Die Schwierigkeiten in der Praxis finden ihren Grund bereits in der Afghanischen Verfassung - AV -. Dreh- und Angelpunkt ist das Spannungsfeld „Religionsklausel (Art. 3 AV) versus völkerrechtliche Verpflichtungen, insb. Menschenrechte (Art. 7 AV)“. Schon dies pflegt als Klassiker in beinahe allen Kursen lebhafte Diskussionen auszulösen. Nach dem Religionsvorbehalt des Art 3 AV ,,darf kein Gesetz der heiligen Religion Islam widersprechen “2 . In der Verpflichtung des Staates zur Beachtung der Allgemeinen Erklärung der Menschenrechte sowie internationaler Verträge, die das Land ratifiziert hat, wird häufig eine Unvereinbarkeit mit den Vorgaben islamischer Normen gesehen und der SchariaVorbehalt stets höher bewertet. Der Scharia-Vorbehalt wird in einer häufig klerikal anmutenden Art und Weise als Totschlag-Argument in den Diskussionen verwendet. Immer wieder flammen heftige Debatten auf zur Gleichheit von Mann und Frau, sowie zur Meinungsfreiheit - obwohl diese Rechte unmittelbar in der Verfassung verankert sind und es keines Rückgriffs auf die völkerrechtlichen Verpflichtungen Afghanistans bedarf, die ohnehin massiver Ablehnung begegnen. Die Kursteilnehmer äußern mit Nachdruck und zum Teil recht aggressiv den Wunsch, sich aus diesen Verpflichtungen zu lösen, ohne jedoch die Beitritts- oder Vorbehaltsmodalitäten im Bereich völkerrechtlicher Verträge auch nur im Ansatz zu kennen. Bemüht man sich um die Erläuterung dieser Modalitäten, stößt dies auf noch vehementeren Widerstand. Diese Schwierigkeiten der Praxis kommen nicht zuletzt dadurch zustande, dass die Verfassung ihr Verhältnis zum Völkerrecht nicht näher bestimmt. Das Schweigen der Verfassung hierüber macht eine völkerrechtsfreundliche Auslegung im Unterricht schwierig bis unmöglich, jedenfalls nur auf das Referieren der Möglichkeiten de lege ferenda begrenzt.

Insgesamt ist festzuhalten: Das Verfassungsverständnis der afghanischen Juristen beschränkt sich vielfach auf das Hervorheben des Scharia-Vorbehaltes in Art. 3 und zwar jenseits der afghanischen Dogmatik, geschweige denn im Rahmen des Völkerrechts. Bei aller Kritik ist allerdings hervorzuheben, dass die Durchsetzung der Interessen ausländischer Staaten, insbesondere USA, eine große Rolle bei der Gestaltung der jetzigen Verfassung, insbesondere des Scharia-Vorbehaltes gespielt hat. So ist von Fachleuten, welche der Verfassungskommission zur Ausarbeitung der afghanischen Verfassung von 2004 beige-

Einen vorzüglichen Überblick über die afghanische Verfassung und deren Verhältnis zum Völkerrecht gibt Michael Schoiswohl, Wo Theorie zur Praxis wird: Internationales (Verfassungs-) Recht und das Entstehen einer Verfassung für das Nachkriegs-Afghanistan, in: Eberhard/Lachmayer/ Thallinger (Hrsg.), Reflexionen zum Internationalen Verfassungsrecht, Wien, 2005, S. 21-47. 
wohnt haben, zu hören, dass jener Scharia-Vorbehalt eine qualitative Verschärfung erst erfahren hat, nachdem die USA auf dem Modell einer Zentralregierung bestanden.

\section{Kollisionen zwischen Gesetz, Scharia und Stammestradition}

Allerorts hervorgehoben wird der normative Pluralismus in Afghanistan. Das klingt zunächst vielversprechend, sind doch damit gemeint das staatlich gesetzte Recht, die Scharia sowie tribale Stammesregeln zur Konfliktbeilegung wie etwa das Pashtunwali. Die Bezeichnung als „,normatives Patchwork“ ${ }^{3}$ vernachlässigt jedoch, dass diese unterschiedlichen Elemente nicht immer auf eine Zusammenarbeit im Sinne der Sache ausgerichtet sind. Selbst da, wo eine Ergänzung von Verfassung wegen vorgesehen ist, wird dies aus vermeintlich religiösen, jedoch nicht näher ausgeführten religionsrechtlichen Argumenten, abgelehnt, wie oben angedeutet. Fakt ist: Die historisch wie kulturell unterschiedlich geprägten Normkomplexe machen außenstehenden Juristen die Arbeit in Afghanistan schwer; einem Teil der afghanischen Juristen eröffnen sie sogar unsägliche Möglichkeiten zu Ausflüchten, um zu gewünschten, weniger rechtsstaatlich orientierten Ergebnissen zu gelangen. Jedenfalls aus der unmittelbaren praktischen Arbeit mit afghanischen Juristen gibt es eher ein Nebeneinander der sich zum Teil widersprechenden Rechtsregime, die sich allenfalls in den konservativen Ansichten beispielsweise zu den Frauenrechten, nur graduell unterscheiden, sich über- oder unterbieten. Und: Ganz gewiss gerät dieser Normenpluralismus in teilweise erhebliche Widersprüche zu den völkerrechtlichen Verpflichtungen Afghanistans.

Aufgrund der rein wissenschaftlichen Expertise des MPI finden die für das Verständnis der afghanischen Gesellschaft und Kultur sehr wichtigen Stammestraditionen, ähnlich wie das religiöse Recht der Schuras (religiöse Räte), im Rahmen der MPI-Kurse keine Auseinandersetzung. Wohl aber ist man mit der Scharia konfrontiert, wobei das Spannungsfeld zwischen ihr und dem staatlich gesetzten Recht immens ist. Scharia hat einerseits Verfassungsrang, ist aber zugleich durch dieselbe begrenzt. Letzteres ist bei den Juristen jedoch weniger bekannt. Die Juristen kommen häufig nicht über die Hausnummer 3 der Verfassung hinaus. Tun sie es doch und erkennen sie, dass die Verfassung in Art. 130 die Subsidiarität des religiösen Rechts (im Falle der Ermangelung von Gesetzen) postuliert, kennen sie die Subsidiaritätsvoraussetzungen der Verfassung nicht. Nach Art. 130 AV müssen Gerichte nämlich dann, ,,wenn in einem zur Entscheidung anstehenden Fall in der Verfassung und den sonstigen Gesetzen keine Bestimmungen zu finden sind, ihre Urteile innerhalb der Grenzen dieser Verfassung in Übereinstimmung mit der hanafitischen Rechtslehre so fällen, dass der Gerechtigkeit auf bestmögliche Weise gedient ist“. Dass beispielsweise die Achtung der Menschenrechte eine solche Grenze der Scharia-Anwendung darstellt, wird einhellig scharf abgelehnt. Paradebeispiel und Stein des Anstoßes ist hierbei sehr oft 
die Problematik der Apostasie. Heftige Diskussionen prägen die Debatten um die Subsidiaritätsregel des Art. $130 \mathrm{AV}$, welche sogar so weit reichen, auch muslimische Dozenten als Ungläubige zu bezeichnen. Das dort verankerte Kollisionsregime wird beispielsweise als Legitimation der Strafe für die Apostasie angeführt. Mit Bezug auf das Gerechtigkeitserfordernis wird, erstaunt über die Norm, vorgebracht, dass Scharia nur gerecht sein könne. Gleichwohl: Macht man die vermeintlichen Experten des religiösen Rechts darauf aufmerksam, dass von den möglichen Auslegungen im hanafitischen Recht die gerechteste zu wählen sei, so wird dies kampflos akzeptiert.

Als wäre das alles nicht schon kompliziert genug, haben staatliche Justiz, religiöse Räte sowie tribale Gerichte eine zusätzliche Konkurrenz zur Seite gestellt bekommen: Die faktische Herrschaft der Taliban in Teilen des Landes hat dort mobile Taliban-Gerichte ermöglicht. Dass die dort gefällten Entscheidungen auf der Grundlage einer orthodoxen Auslegung der Scharia getroffen werden, mag angesichts der Geschichte der Taliban in diesem Land niemanden verwundern. Die Arbeit der staatlichen Justiz und die Entwicklung der rechtsstaatlichen Elemente in Afghanistan werden zur Farce.

Festzuhalten bleibt: Die drei Regelungssysteme werden von mannigfachen Akteuren vertreten, fortentwickelt und von konkurrierenden Institutionen angewendet, mit all den damit einhergehenden Widersprüchen und Rechtshemmnissen. Eine vermeintlich gegenseitige Ergänzung der Normen ist daher vielfach eher theoretisches Wunschdenken als rechtliche Realität.

\section{Schlussanmerkungen}

Im Bereich der afghanischen Justiz eine positive wie kontinuierliche Entwicklung zu diagnostizieren wäre alles andere als redlich. Die Justiz ist ein Spiegelbild der gegenwärtigen Lage in Afghanistan: gespalten, strukturlos und anarchisch. Sie wird durch die skizzierten inhaltlichen wie auch praktischen Probleme massiv belastet. Es gibt insbesondere in der Provinz aufgrund fehlender Urbanität keinen zumutbaren Zugang zu staatlichen Gerichten. Findet der Bürger endlich - im wahrsten Sinne des Wortes - den Weg zum Recht, so stärken langwierige Prozesse sowie Korruption im Justizsektor nicht gerade das Vertrauen der Bevölkerung in denselben. Dem afghanischen Juristen Ali Wardak zufolge hat sich sogar eine Kultur der Menschenrechtsverletzung etabliert, die teilweise auch vor einigen internationalen „Helfern“ nicht halt macht - letzteres ist freilich Wasser auf die Mühlen der Geistlichen, die die Bevölkerung stets vor den internationalen Gremien ,warnen“, so einige afghanische Richter hinter vorgehaltener Hand.

Doch Fundamentalkritik ist nicht am Platz. Vielmehr sollten die historischen Gegebenheiten Afghanistans betrachtet werden. Noch bis Anfang der 1970er Jahre war die Scharia die Grundlage der Rechtsordnung, bis eine moderne Justiz mit Staatsanwaltschaft und Oberstem Gerichtshof etabliert wurde. Justiz und Gerichtsbarkeit wurden unter den Taliban durch das Amt für die „Förderung der Tugend und Bekämpfung des Lasters“ mit seiner rückwärtsgewandten und menschenverachtenden Rechtspraxis ersetzt. Erstmals verbindet 
also die Verfassung der Islamischen Republik Afghanistan vom Januar 2004 die Werte des Islam mit der Demokratie. Die Kehrseite ist: Der Versuch, Tradition und Moderne zu versöhnen, hat sich unter den stark religiösen Vorstellungen als ein handfester Widerspruch erwiesen, der große Probleme in der Rechtspraxis aufwirft, etwa im Hinblick auf die Menschenrechtsgewährleistungen. Die gesellschaftliche Wirklichkeit ist von den Verfassungsgarantien, etwa der Gleichheit von Mann und Frau, nicht nur weit entfernt, sie ist weitgehend konträr dazu.

Wenn man nun fairerweise nicht außer Acht lässt, dass das Land gerade einmal sieben Jahre mit dem Prozess dieser Entwicklung befasst ist, fällt das Urteil wiederum etwas milder aus. Doch auch dann, wenn man die Entwicklung des Rechtsstaates als einen langwierigen Prozess kleiner und nur manchmal größerer Schritte begreift, so fällt bei allem Verständnis für die enormen Probleme des Landes auf, dass selbst die kleinen Schritte sehr zäh verlaufen. Die Hemmnisse scheinen größer und stabiler als der Fortschritt. Während die Afghanen die Justizaufbauprojekte zwar grundsätzlich begrüßen, stehen sie ihnen aber auch sehr skeptisch gegenüber. Eine „Wasch mir den Pelz, aber mach mich nicht nass-Mentalität" ist sehr oft anzutreffen. Darüber hinaus ist die Korruption ein zentrales Problem bei einer fundamentalen Säule der Rechtsstaatlichkeit, nämlich der Unabhängigkeit der Justiz, gefolgt von organisatorischen und technischen Hindernissen. Dies wurde in der unmittelbaren Arbeit mit afghanischen Richtern und Staatsanwälten vor Ort in unfassbaren Geschichten immer wieder referiert. Im Kern ging es bei den Erzählungen darum, dass die Richter so oder so in einem Dilemma stecken: Sie sind entweder wegen der Verzweiflung um die Versorgung ihrer Familie, oder aber angesichts der Karrierechancen bzw. drohenden Jobverlusts bis hin zur Gefahr für Leib und Leben der Bestechlichkeit verfallen, weshalb sie entsprechende Anweisungen nicht ablehnen (können). Noch unfassbarer ist allerdings die Tatsache, dass alte Kriegsherren häufig noch immer in Schlüsselpositionen des Staates tätig sind - alter Wein in neuen Schläuchen. Viele Afghanen beklagen die Strategie, die ehemaligen Warlords ins staatliche Gefüge implementiert zu haben, als kontraproduktiv. Anstelle im Dienste des Volkes zu handeln, habe der Staat den regionalen Warlords und Machthabern zu viele Zugeständnisse gemacht, ihnen so zu neuer Stärke verholfen. Und dies obwohl viele Verbrechen der Vergangenheit auf ihr Konto gehen. Dadurch habe das Vertrauen der Bevölkerung in eine unabhängige und gerechte Justiz stark gelitten, was zur Abkehr der Bevölkerung von der Justiz und zur Stärkung von außerstaatlichen Streitbeilegungsmechanismen geführt habe. Wie entsprechende Maßnahmen zur Bekämpfung der Korruption in der Afghan National Development Strategy (ANDS) wirken, bleibt abzuwarten. Doch nicht einmal zu verhaltenem Optimismus sind üppige Gründe vorhanden.

In der „Entwicklungssackgasse“ haben viele Afghanen und ihre politischen Helfer eines gemeinsam: Sie müssten erkennen, dass eine Kultur des Unrechtsbewusstseins und der Rechtsstaatlichkeit noch nicht existiert. Es gilt zu erkennen, dass dies kein exportfähiges Gut ist, wenn die dazu korrespondierenden Überzeugungen nicht aus der Mitte der afghanischen Gesellschaft kommen. Es bedarf einer redlichen wie sorgfältigen Generalüberholung der Denkkonzepte aller politisch Beteiligten, also jener der afghanischen Machtherren wie 
auch ihrer politischen Helfer, um die Arbeit von unabhängigen Institutionen, wie etwa des MPI zu effektivieren.

Im Rahmen einer wertenden Gesamtbetrachtung bleibt festzuhalten: Alle kritischen Anmerkungen sollten von der Grundannahme ausgehen, dass eine Verfassung den soziologischen, ethnischen, psychologischen Gegebenheiten eines Landes Rechnung tragen muß. Diese können je nach Kulturkreis unterschiedliche Ausprägen haben und sollen das auch, denn eine Verfassung ist nicht mehr - aber auch nicht weniger - „eine sich aus staatlichen Interpretationen entwickelte und verwirklichte Vernünftigkeit eines Volkes“. ${ }^{4}$ Trotzdem, oder vielleicht gerade deshalb soll versucht werden, Kritik aus jener Warte zu üben, welche sich globalen Menschenrechten verpflichtet fühlt und dennoch ihre Gewährleistungsmöglichkeiten im Kontext der afghanischen Gegebenheiten sucht. Solange muss konstatiert werden, dass Afghanistan trotz des entwicklungs- und sicherheitspolitischen Engagements von zahlreichen Nationen gegenwärtig noch weit von einer stabilen Justiz und somit von einem Rechtsstaat entfernt ist. Denn: In der Umsetzung rechtsstaatlicher Elemente kommt das Verhältnis von Demokratie und Rechtsstaat zum Ausdruck: „Zwischen Rechtsstaat und Demokratie besteht nicht nur ein historisch-zufälliger, sondern ein begrifflicher oder interner Zusammenhang" ${ }^{5}$ - Die Stammeskultur Afghanistans hat hier noch einen steinigen Weg vor sich. 